\title{
Empyema Necessitans in the Setting of Methicillin-Susceptible Staphylococcus aureus Causing Pneumonia and Bacteremia
}

\author{
Sindhura Bandaru $\mathbb{D}^{1},{ }^{1}$ Sukesh Manthri $\mathbb{D}^{2},{ }^{2}$ Vidya Sundareshan, ${ }^{1}$ and Vidhya Prakash $\mathbb{D D}^{1}$ \\ ${ }^{1}$ Southern Illinois University School of Medicine, Springfield, IL, USA \\ ${ }^{2}$ Saint Louis University, St. Louis, MO, USA \\ Correspondence should be addressed to Vidhya Prakash; vprakash59@siumed.edu
}

Received 8 February 2018; Accepted 25 March 2018; Published 5 April 2018

Academic Editor: Gernot Walder

Copyright ( $) 2018$ Sindhura Bandaru et al. This is an open access article distributed under the Creative Commons Attribution License, which permits unrestricted use, distribution, and reproduction in any medium, provided the original work is properly cited.

\begin{abstract}
Empyema necessitans (EN) is a rare phenomenon that refers to an insidious extension of the empyema through parietal pleura and subsequent dissection into subcutaneous tissue of the chest wall. A 29 -year-old man presented to the hospital with fever and chills a few days after an inadvertent needle stick while injecting heroin. His left forearm was warm with an area of fluctuance. He underwent incision and drainage of the left forearm abscess with fluid submitted for Gram stain and culture. His condition rapidly deteriorated due to sepsis, and he required transfer to the intensive care unit. A new $4 \times 3 \mathrm{~cm}$ area over the left pectoralis muscle had become increasingly indurated, fluctuant, and erythematous. CT of the chest demonstrated extensive cavitary lung lesions and a large loculated left-sided pleural effusion with extension through the chest wall. TEE revealed a $3 \mathrm{~cm}$ complex lesion on the superior septal leaflet of the tricuspid valve. The patient underwent incision and drainage of the pectoralis major EN with placement of a drain. Blood and sputum cultures grew methicillin-susceptible Staphylococcus aureus (MSSA) at which time antibiotic therapy was tailored to oxacillin. Our case highlights a rare occurrence of EN due to MSSA in a patient with intravenous drug use (IDU) and underscores the importance of prompt diagnosis and treatment.
\end{abstract}

\section{Introduction}

Empyema is a collection of pus in the pleural space. It is commonly caused by certain bacterial pathogens and requires drainage in addition to appropriate antibiotics for definitive therapy. Empyema necessitans $(\mathrm{EN})$ is a rare entity that refers to an insidious extension of the empyema through parietal pleura and dissection into the subcutaneous tissue. EN typically occurs in the setting of long-standing parapneumonic effusions, especially those that are tuberculous in nature. It rarely presents secondary to an acute bacterial infection. We report a rare case of EN due to MSSA occurring acutely in a patient with intravenous drug use (IDU).

\section{Case Report}

We describe a case of a 29-year-old man with a history of IDU who presented to the hospital with fever and chills a few days after an inadvertent needle stick while injecting heroin.
Avoiding medical care at first, he eventually came to the Emergency Department when he developed swelling around the punctured skin over the inferior aspect of the left cubital fossa. Upon initial evaluation, he had a temperature of $37.3^{\circ} \mathrm{C}$, heart rate of 104 per minute, and respiratory rate of 38 per minute. The inferior aspect of the left cubital fossa was warm, erythematous, and with a $3.5 \mathrm{~cm} \times 3.5 \mathrm{~cm}$ wellcircumscribed area of fluctuance and induration. Laboratory evaluation revealed a hemoglobin of $10.9 \mathrm{gm} / \mathrm{dl}$ and white blood cell count of $10.6 \mathrm{k} / \mathrm{cumm}$ with a neutrophilic predominance but no immature granulocytes. His absolute neutrophil count was $9.1 \mathrm{k} /$ cumm (normal 1.5-6.5 k/cumm). The comprehensive metabolic panel showed normal renal function and transaminases but elevated total bilirubin $(4.5 \mathrm{mg} / \mathrm{dl})$. His chest radiograph showed patchy infiltrates throughout the mid and upper right lung field with possible small right pleural effusion. Blood and sputum cultures were ordered, and he was started on broad-spectrum antibiotics consisting of vancomycin $1.5 \mathrm{gm}$ IV every 8 hours 


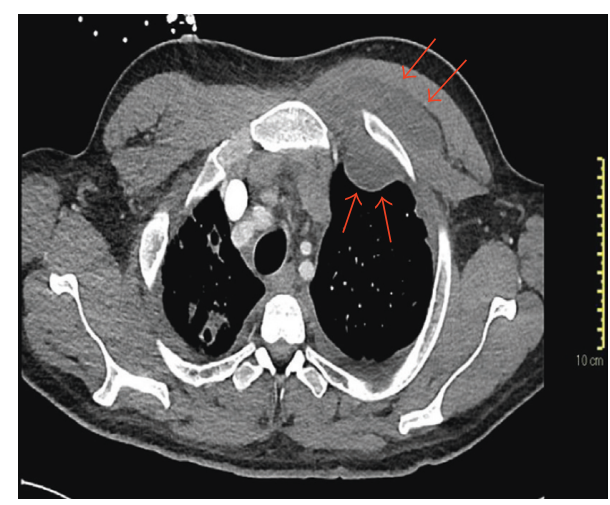

Figure 1: Multiple cavitary nodules within the right lung apex representing septic emboli. Bilateral pleural effusions with left-sided pleural-based focus of confluent fluid attenuation that extends through the anterior chest wall and insinuates between the pectoralis major and minor muscles representing empyema necessitans (arrows) are shown.

$(\sim 15 \mathrm{mg} / \mathrm{kg})$, piperacillin-tazobactam $4.5 \mathrm{gm} \mathrm{IV} \mathrm{every} 8$ hours given as an extended infusion, and levofloxacin $750 \mathrm{mg}$ IV once daily. He underwent incision and drainage of the cubital abscess. His antibiotics were tailored to oxacillin 2 gm IV every 4 hours as blood and sputum cultures revealed growth of methicillin-susceptible Staphylococcus aureus. On hospital day 6 , his condition rapidly deteriorated due to sepsis (persistent fevers, tachycardia, and hypotension with rising white blood cell count), and a new $4 \mathrm{~cm} \times 3 \mathrm{~cm}$ area of fluctuance was noticed on the left superior pectoralis region. He was transferred to the intensive care unit. A transesophageal echocardiogram (TEE) revealed a multilobed lesion on the superior and septal leaflets of the tricuspid valve, with lesion dimension approximating $3 \mathrm{~cm}$ on the superior cusp. CT of the chest with intravenous contrast demonstrated extensive bilateral cavitary lung lesions likely reflecting septic emboli, reactive mediastinal and hilar lymphadenopathy, and a large left-sided pleural effusion. A loculated component along the left upper lung insinuated through the chest wall into the left pectoralis muscle, raising the possibility of empyema necessitans (Figures 1 and 2).

Incision and drainage was performed with subsequent indwelling catheter placement for drainage of pectoralis major EN. For the left-sided pleural effusion, a chest tube was placed and it drained serosanguinous fluid. The cardiothoracic surgery service evaluated the patient and recommended ongoing antibiotic therapy with close clinical and radiographic surveillance. CT of the chest, when repeated, showed no interval change in multiple bilateral cavitary lesions, and a surveillance TEE done two weeks later identified persistent tricuspid vegetations with minimal regurgitation. As the $\mathrm{CT}$ of the chest was stable and the patient was clinically improving, surgery to replace the tricuspid valve was deferred with plans to treat the patient with six weeks of oxacillin.

The patient completed six weeks of intravenous oxacillin and was later started on suppressive antibiotic therapy with oral dicloxacillin $500 \mathrm{mg}$ twice daily given the fact that he had high disease burden and had not undergone surgical intervention. Follow-up transesophageal echocardiogram (TEE)

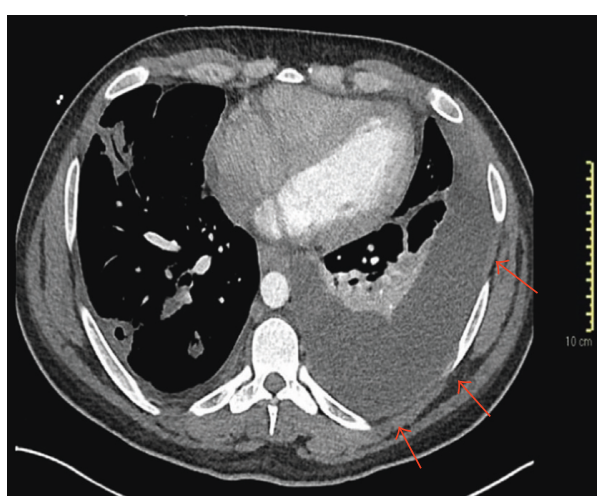

FIgURE 2: Arterial-phase axial computed tomographs of the lower thorax demonstrating a moderate left pleural effusion with associated compressive atelectasis. Peripheral cavitary pulmonary lesions and partially visualized tricuspid valve (with known vegetation) are shown.

done soon after completing six weeks of antibiotic treatment showed that the tricuspid valve lesions had stabilized, if not minimally regressed in size, but tricuspid insufficiency had progressed to a moderate range. Magnetic resonance (MR) cardiac imaging reconfirmed sclerosis/thickening of the tricuspid valve leaflets, with moderate-to-severe tricuspid valve regurgitation. Surveillance radiography with CT of the chest showed that the cavitary lesions throughout the lungs had resolved. Unfortunately, at this point, the patient was lost to follow-up.

\section{Discussion}

Empyema necessitans (EN) refers to extension of a pleural infection out of the thorax and into the surrounding soft tissue of the chest wall and other neighboring structures. The exact pathophysiology is unclear. It may either occur in the setting of previous thoracic surgery (e.g., thoracotomy) or trauma or result from inadequate treatment of an empyema, typically occurring after a necrotizing pneumonia or pulmonary abscess [1].

The current literature notes that Mycobacterium tuberculosis accounts for approximately $70 \%$ of cases of EN [2]. Actinomyces is considered the second most common cause $[3,4]$. Cases of EN due to fungal pathogens such as Blastomyces, Aspergillus species, and Mucormycosis have been described less frequently. EN due to MSSA in an immunocompetent host resulting from IDU has not previously been reported, although there have been published reports of EN due to other bacteria including MRSA, Fusobacterium, and Nocardia $[5,6]$.

Sharma and Blyth [7] reported an unusual case of ruptured lung abscess, complicated by a persistent air leak and EN caused by Bacteroides species. However, Bacteroides is a common cause of intrapulmonary abscess and pleural infection. Yauba et al. [8] described a pediatric case of EN due to Proteus species and discussed challenges in diagnosis and management as it was difficult to differentiate between tuberculous and nontuberculous effusions.

Staphylococcus aureus (S. aureus) is the most common cause of infective endocarditis (IE) in much of the developed 


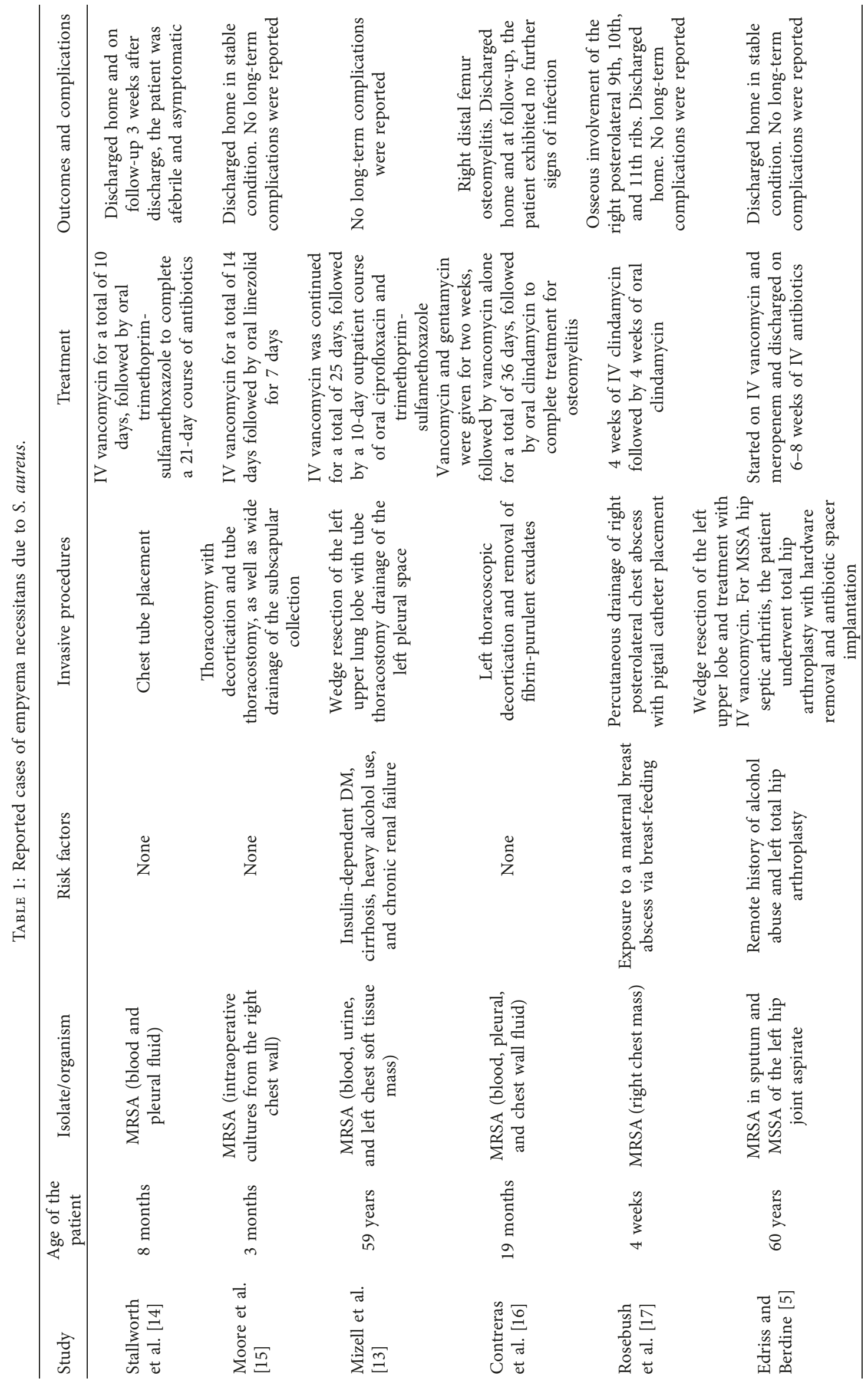


world. Data from $>70$ million hospitalizations in the United States suggest that rates of $S$. aureus IE have increased significantly relative to other causes of IE [9]. S. aureus IE in patients with IDU often involves the tricuspid valve. Cure rates for right-sided $S$. aureus IE in IDU are high (>85\%) and may be achieved with relatively short courses of either parenteral or oral treatment [10]. Complicated IE manifested by deep tissue abscesses or osteoarticular infection may require prolonged therapy. S. aureus IE complicated by EN is not as clearly delineated in literature, but we considered this as a complication of IE involving the tricuspid valve.

In adults, EN due to Streptococcus pneumoniae has rarely been reported [11], which is a similar trend for EN due to $S$. aureus infection [12]. Table 1 shows all reported cases of EN due to $S$. aureus, including pediatric cases compiled after a literature search in PubMed. Edriss and Berdine [5] described a case of EN secondary to methicillin-resistant $S$. aureus (MRSA). This patient developed a rounded painful swelling of the left upper chest after he tripped and fell. Mizell et al. [13] also described a case of EN secondary to MRSA in a patient with diabetes and cirrhosis. Unlike this case, our patient did not have any risk factors such as immunosuppression, diabetes, chest trauma, or thoracic surgery. We theorize that our patient's infection started as a skin and soft tissue infection due to MSSA in the left antecubital fossa with subsequent bacteremia and seeding of his tricuspid valve, followed by embolic phenomenon to his lungs which led to a parapneumonic effusion and ultimately direct extension through the chest wall to the pectoralis muscle, as seen in Figure 1. EN commonly spreads to the subcutaneous tissues of the chest wall and can also involve spread to other sites such as the esophagus, breast, retroperitoneal, peritoneal, pericardial, and paravertebral regions. The resultant subcutaneous abscess may eventually rupture through the skin [13].

In terms of diagnosing $\mathrm{EN}$, plain radiographs are often nonspecific and at times can even be normal. At best, plain radiographs may suggest a soft tissue density in the chest wall. Chest CT is best at assessing the extent of infection out of the thoracic cavity. Chest CT will classically reveal an empyema (often a relatively well-demarcated collection) with extension through the chest wall into an extrathoracic compartment. Accompanying rib destruction may be present [5].

Management options for EN include drainage (open or closed) of the pleural space to expand the lung and mitigate risks of fibrosis. Appropriate antibiotic therapy is also a mainstay of treatment [4]. The addition of gentamicin to nafcillin or oxacillin was previously recommended for treatment of complicated right-sided IE due to MSSA. However, recent data suggest that the risks of nephrotoxicity from gentamicin outweigh the benefits of adjunctive antibiotic therapy in IE from S. aureus. Therapy for MSSA IE in patients unable to tolerate $\beta$-lactams is problematic. For patients with a well-defined history of nonanaphylactoid reactions to penicillins (e.g., simple skin rash), a firstgeneration cephalosporin such as cefazolin may be a reasonable choice, and vancomycin is often recommended as an alternative to $\beta$-lactam therapy for patients with anaphylactoid reactions. $\beta$-lactam allergy evaluation is recommended in cases where the nature of the allergy is not well defined, particularly due to the association of poor outcomes with vancomycin for infections due to MSSA. For patients with complications of IE such as perivalvular abscess formation and septic emboli, therapy with nafcillin or oxacillin for at least 6 weeks is warranted [18].

Our case highlights the management principles for all cases of EN, which include appropriate antibiotic therapy and management of the empyema that extends to surrounding tissue, which involves placement of an indwelling catheter and facilitates drainage and lung reexpansion.

In conclusion, EN due to $S$. aureus is an uncommon infection in healthy adults without comorbidities. EN has been associated with complications of thoracotomy, immunosuppressed status, or trauma, none of which applied to our patient. Although there have been rare reports of MRSA as a cause of EN in pediatric and adult populations, MSSA has not, to our knowledge, been reported in the literature. It is difficult to ascertain the exact reason why the pathogen in our case was MSSA as opposed to MRSA. We consider this case to have a rare presentation of EN resulting from an acute complication of MSSA pneumonia resulting from septic emboli in an immunocompetent host.

\section{Conflicts of Interest}

The authors declare that there are no conflicts of interest regarding the publication of this article.

\section{References}

[1] S. I. Ahmed, R. E. Gripaldo, and O. A. Alao, "Empyema necessitans in the setting of pneumonia and parapneumonic effusion," American Journal of the Medical Sciences, vol. 333, no. 2, pp. 106-108, 2007.

[2] J. A. Choi, K. T. Hong, Y. W. Oh et al., "CT manifestations of late sequelae in patients with tuberculous pleuritis," American Journal of Roentgenology, vol. 176, no. 2, pp. 441-445, 2001.

[3] G. F. Mabeza and J. Macfarlane, "Pulmonary actinomycosis," European Respiratory Journal, vol. 21, no. 3, pp. 545-551, 2003.

[4] W. R. Webb and S. S. Sagel, "Actinomycosis involving the chest wall: CT findings," American Journal of Roentgenology, vol. 139, no. 5, pp. 1007-1009, 1982.

[5] H. Edriss and G. Berdine, "Empyema necessitatis secondary to Staphylococcus aureus lung abscess," Southwest Respiratory and Critical Care Chronicles, vol. 5, no. 20, p. 58, 2017.

[6] M. L. Hockensmith, D. L. Mellman, and E. L. Aronsen, "Fusobacterium nucleatum empyema necessitans," Clinical Infectious Diseases, vol. 29, no. 6, pp. 1596-1598, 1999.

[7] V. Sharma and K. G. Blyth, "Empyema necessitans and a persistent air leak associated with rupture of an anaerobic lung abscess due to Bacteroides," Thorax, vol. 73, no. 1, pp. 91-93, 2018.

[8] M. S. Yauba, H. Ahmed, I. A. Imoudu, M. O. Yusuf, and H. U. Makarfi, "Empyema necessitans complicating pleural effusion associated with Proteus species infection: a diagnostic dilemma," Case Reports in Pediatrics, vol. 2015, Article ID 108174, 4 pages, 2015.

[9] J. J. Federspiel, S. C. Stearns, A. F. Peppercorn, V. H. Chu, and V. G. Fowler Jr., "Increasing US rates of endocarditis with Staphylococcus aureus: 1999-2008," Archives of Internal Medicine, vol. 172, no. 4, pp. 363-365, 2012. 
[10] A. W. Heldman, T. V. Hartert, S. C. Ray et al., "Oral antibiotic treatment of right-sided staphylococcal endocarditis in injection drug users: prospective randomized comparison with parenteral therapy," American Journal of Medicine, vol. 101, no. 1, pp. 68-76, 1996.

[11] Y. Ko, K. Tobino, Y. Yasuda, T. Sueyasu, and S. Nishizawa, “A community-acquired lung abscess attributable to Streptococcus pneumoniae which extended directly into the chest wall," Internal Medicine, vol. 56, no. 1, pp. 109-113, 2017.

[12] L. A. Chaudhry, A. A. Ba, M. Zamzami, and A. A. Robert, "Contemporary empyema thoracis necessitans in an adult male caused by Staphylococcus aureus: decortication is superior to traditional under water seal intercostal tube in chronic empyema," Pan African Medical Journal, vol. 20, p. 115, 2015.

[13] K. M. Mizell, K. V. Patterson, and J. E. Carter, "Empyema necessitans due to methicillin-resistant Staphylococcus aureus: case report and review of the literature," Journal of Clinical Microbiology, vol. 46, no. 10, pp. 3534-3536, 2008.

[14] J. Stallworth, E. Mack, and C. Ozimek, "Methicillin-resistant Staphylococcus aureus empyema necessitatis in an eightmonth-old child," Southern Medical Journal, vol. 98, no. 11, pp. 1130-1131, 2005.

[15] F. O. Moore, J. D. Berne, T. M. McGovern, S. Ravishankar, N. B. Slamon, and J. H. Hertzog, "Empyema necessitatis in an infant: a rare surgical disease," Journal of Pediatric Surgery, vol. 41, no. 7, pp. E5-E7, 2006.

[16] G. A. Contreras, N. Pérez, J. R. Murphy, T. G. Cleary, and G. P. Heresi, "Empyema necessitans and acute osteomyelitis associated with community acquired methicillin resistant Staphylococcus aureus in an infant," Biomédica, vol. 29, no. 4, pp. 506-512, 2009.

[17] J. Rosebush, R. Summers, J. Snitzer, R. Jerris, S. Satola, and P. Spearman, "Methicillin-resistant Staphylococcus aureus empyema necessitatis in a breast-fed neonate," Pediatric Infectious Disease Journal, vol. 33, no. 6, pp. 668-669, 2014.

[18] L. M. Baddour, W. R. Wilson, A. S. Bayer et al., "Infective endocarditis in adults: diagnosis, antimicrobial therapy, and management of complications: a scientific statement for healthcare professionals from the American Heart Association," Circulation, vol. 132, no. 15, pp. 1435-1486, 2015. 


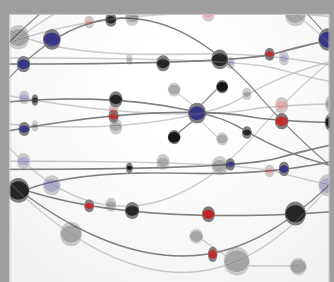

The Scientific World Journal
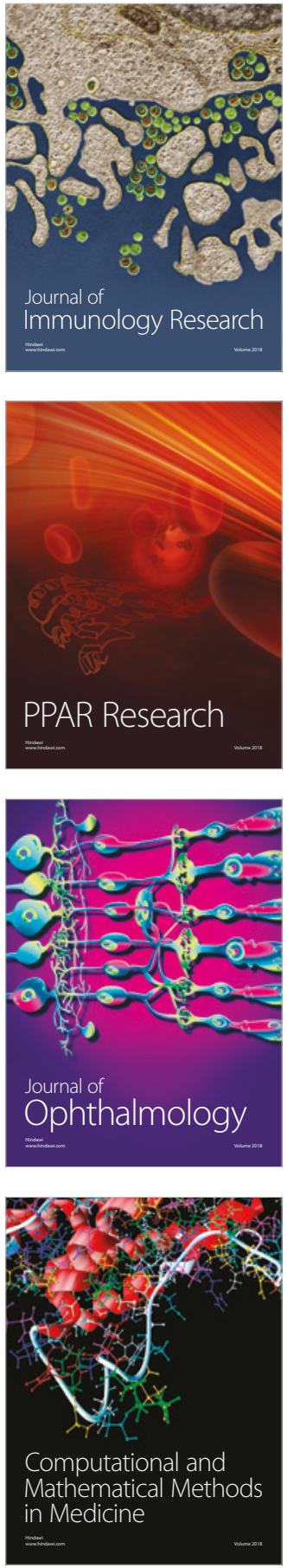

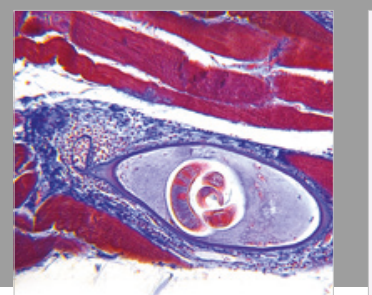

Gastroenterology Research and Practice

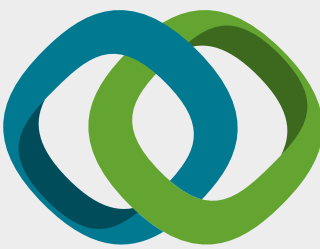

\section{Hindawi}

Submit your manuscripts at

www.hindawi.com
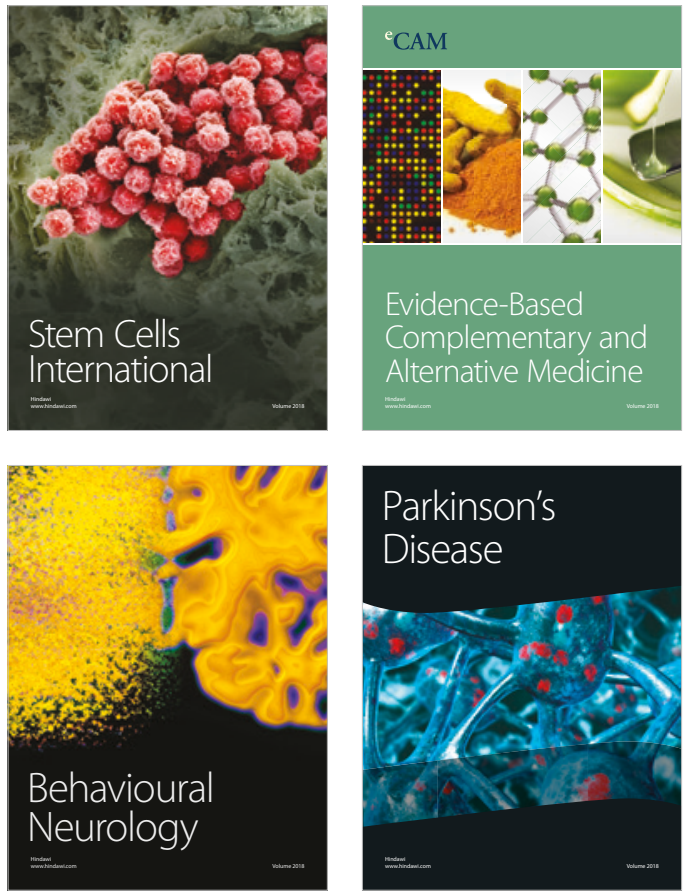

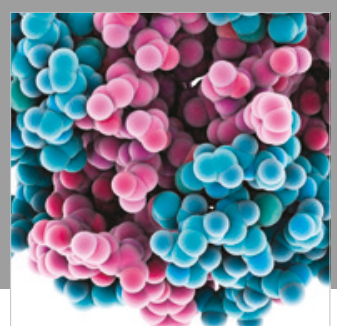

ournal of

Diabetes Research

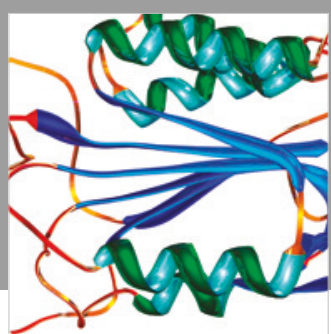

Disease Markers
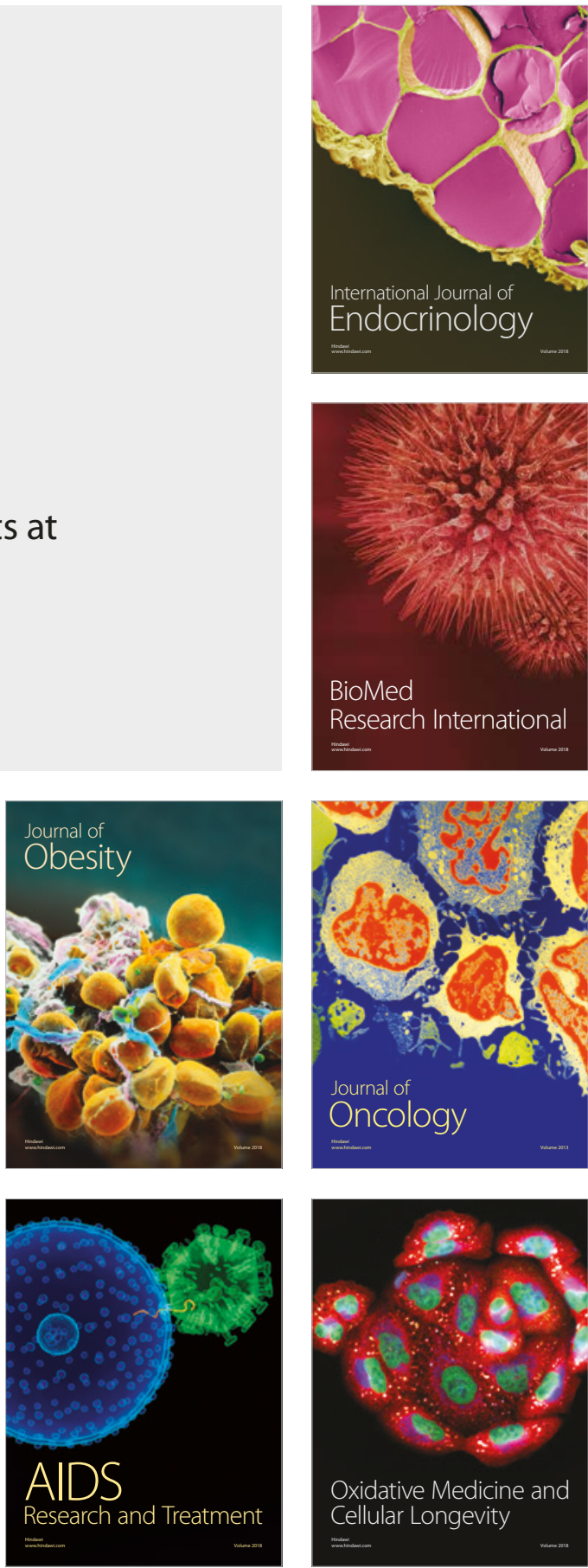